Article type : Clinical Review Article

Short title running head: Diagnosis of scabies

Authors running head: $M$. J. Thompson et al.

Running section head: Clinical dermatology

Correspondence: Assistant Professor Andrew Steer, Centre for International Child Health, Royal Children's Hospital Melbourne, Flemington Road, Parkville, Victoria, 3052, Australia

E-mail: andrew.steer@rch.org.au

Conflict of interest: none declared

Accepted for publication 18 September 2016

Review article

\title{
Systematic review of the diagnosis of scabies in therapeutic trials
}

\author{
M. J. Thompson, ${ }^{1}$ D. Engelman, ${ }^{2,3}$ K. Gholam ${ }^{4}$, L. C. Fuller ${ }^{5,6}$ and A. C. Steer ${ }^{2,3}$ \\ ${ }^{1}$ Department of Paediatrics and Child Health, Western Australia Country Health Service, Kimberley Region, \\ Australia; ${ }^{2}$ Department of Paediatrics, Centre for International Child Health, University of Melbourne, Melbourne, \\ Australia; ${ }^{3}$ Group A Streptococcal Research, Murdoch Childrens Research Institute, Melbourne, Australia; ${ }^{4}$ Dermatology \\ Department, Great Ormond Street Hospital, London, UK; ${ }^{5}$ International Foundation for Dermatology, London, UK; \\ and ${ }^{6}$ Department of Dermatology, Chelsea and Westminster NHS Foundation Trust, London, UK
}

\section{Summary}

Human scabies (infestation with the mite Sarcoptes scabiei var hominis) causes a significant disease burden worldwide, yet there are no agreed diagnostic guidelines. We aimed to determine whether a consistent approach to diagnosing scabies has been used for published scabies therapeutic trials. The data sources used were the MEDLINE, Embase and Cochrane databases, from 1946 to 29 August 2013. Eligible studies were trials of therapeutic interventions against scabies in human subjects, published in English, enrolling patients with scabies, and using various therapeutic interventions. It should be noted that language was a limitation of this study, as some relevant trials published in languages other than English may have been excluded. Each study was reviewed by two independent authors, who assessed the clinical examination and testing approaches used for scabies diagnosis used in the included studies. Summary statistics of trial settings and diagnostic approaches were calculated, with associations examined using $\chi^{2}$ testing. We found that of 71 included trials, 40 (56\%) specified which clinical findings were used for This is the author manuscript accepted for publication and has undergone full peer review but has not been through the copyediting, typesetting, pagination and proofreading process, which may lead to differences between this version and the Version of Record. Please cite this article as doi: $10.1111 /$ ced.13152

This article is protected by copyright. All rights reserved 
diagnosis, which were predominantly rash, rash distribution, pruritus and mite burrows. Parasitological testing was used in $63 \%$ of trials $(n=45)$ and was used more frequently in clinic-based than in field studies. Nearly one-quarter of trials $(24 \%, n=17)$ did not define the diagnostic method used. Overall, the diagnostic approaches were poorly described, prohibiting accurate comparison of existing studies. This review further supports the need for consensus diagnostic guidelines for scabies.

\section{Introduction}

Human scabies is caused by infestation with the mite Sarcoptes scabiei var hominis, and is underrecognised as a public health problem, despite generating a considerable global disease burden, affecting over 100 million people., ${ }^{1,2}$ Scabies predominantly affects marginalized groups and those that experience poverty and overcrowding, ${ }^{2}$ perpetuating inequity for vulnerable populations. ${ }^{3}$ Prevalence varies widely between and within populations, ${ }^{4}$ with high rates in the Pacific and up to $78 \%$ of young children affected in one area of Central America. ${ }^{5,6}$ Confined residential settings such as orphanages, nursing homes, displacement camps or prisons may generate epidemics, when peak prevalence may reach over $70 \%{ }^{7,8}$

The itch caused by scabies has a direct effect on quality of life, causing sleep loss and subsequent reduced productivity. ${ }^{9}$ Scabies is associated with bacterial skin infection, predominantly with Staphylococcus aureus and Streptococcus pyogenes, which in turn can lead to invasive bacterial disease. ${ }^{10,11}$ There are clear links between scabies and acute post-streptococcal glomerulonephritis, ${ }^{10,11}$ and there are emerging epidemiological links between scabies-associated pyoderma and acute rheumatic fever. ${ }^{12}$ Hyperinfestation (crusted or Norwegian scabies), usually in the setting of immunological compromise, is associated with high mortality. ${ }^{13}$

Although there are a few national guidelines and diagnostic algorithms, there are no internationally agreed standards for the diagnosis of scabies infestation, making epidemiological and therapeutic studies difficult to interpret and compare. The presence of only 10-15 adult female mites in classic infestation ${ }^{14,15}$ makes direct observation challenging. Clinical identification of a burrow is pathognomonic, but it may not be present or demonstrable in all cases. ${ }^{16,17}$ Other scabies-associated skin lesions may mimic a wide variety of dermatological conditions. The gold standard of diagnosis is demonstration of a live mite, eggs or faecal material (scybala) via skin scraping methods, but false negatives are common, ${ }^{3}$ and present difficulties in using this test as a comparative tool. Furthermore, such testing requires microscopy, and is not always practicable in all clinical or field epidemiology settings. Dermatoscopy or high-power epiluminescence microscopy has been shown to be an effective adjunct to clinical examination, ${ }^{18,19}$ but has not been widely adopted. Other serological and molecular tests show promise, but they are not yet sufficiently sensitive or widely available to enable regular human use..$^{20,21}$

To enable research collaboration and comparison, a consistent diagnostic approach is required. ${ }^{1}$ We conducted a systematic review of diagnostic methods and criteria used in therapeutic trials for scabies.

\section{Methods}

Using a systematic review approach, we searched for studies of therapeutic trials for scabies (Fig. 1). We reviewed therapeutic trials rather than observational studies because we expected that treatment studies might have a higher level of precision and documentation of diagnostic definitions. Trials were not assessed for bias because outcome measures were not examined.

THE MEDLINE, Embase and Cochrane databases were searched for trials between 1946 and August 
2013, using the terms 'scabies OR sarcoptes' and 'treatment OR therapy'. All abstracts were examined for relevance by one reviewer. Further relevant articles were identified via review of reference lists. Studies that were not therapeutic trials for scabies, were not human trials, or were not available in English were excluded.

Data were extracted from the final set of included articles by two independent reviewers, with any coding conflicts resolved by a third party. Information was recorded about the study population and the country where the trial took place. Trials were defined as clinic-based if they were conducted in an outpatient clinic or within a hospital, and field-based if they were conducted in the community or in an institution; the latter was defined as a camp, school, prison, orphanage, aged care facility or homeless shelter. A resourcelimited country was defined as a country within the lower three categories of the 2013 United Nations Human Development Index. ${ }^{22}$

Data relating to how scabies was diagnosed were extracted from the Methods section of all articles. Rash characteristics and distribution were captured, and trials listing rash distribution as 'classical', 'typical', or 'sites of predilection' without further definition were grouped together under 'classical distribution'. Those trials that did not define the methods used for diagnosing scabies, for example stating, 'patients with scabies were enrolled', were classified as 'unspecified'. The use of magnification was also captured, and included formal epiluminescence microscopy and use of a hand lens.

Data regarding testing for scabies were also extracted from the Methods section of each article. Trials that reported 'parasitological confirmation' without further detail were coded as using testing. A skin scraping was defined as a scraping of the epidermis with a scalpel and microscopy of the resultant sample; studies that described alternative extraction methods were coded separately. The method of sample processing was recorded. Findings for a positive microscopy result were divided into either mite identification (visualization of an adult or larval mite) or product identification (defined as either scybala or mite eggs), or both.

Summary statistics and combinations of clinical diagnosis and testing were calculated using Excel for Mac 2011 (v014.4.3; Microsoft Corp., Redmond, WA, USA). Associations between the trial characteristics and the study's diagnostic approach were examined using $\chi^{2}$ testing (SPSS software v20.0; IBM, Armonk, NY, USA). Our results are reported according to PRISMA guidelines. ${ }^{23}$

\section{Results}

We initially identified 239 articles, with 71 meeting inclusion criteria (Figure 1). Trials were predominantly clinic-based $(n=42,59 \%)$ and just over half the trials included all age groups ( $n=37,52 \%$; Table 1$)$. Three-quarters of the trials were conducted in resource-limited countries $(n=53)$, with $55 \%$ of these in a field setting $(n=29)$

Only 40 trials $(56 \%)$ specified what clinical findings were used for diagnosis of scabies (Table 2). Of these, the predominant features mentioned as part of the diagnostic process were rash $(n=40,100 \%)$, rash distribution ( $n=30,75 \%)$, pruritus $(n=28,70 \%)$ and mite burrows $(n=22,55 \%)$. Magnification was used to assist diagnostic examination in only 5 of all 71 trials $(7 \%)$.

A number of studies required several clinical features to be present for a diagnosis to be made, with up to 12 characteristics of rash appearance and distribution detailed in one study. ${ }^{24}$ Although $23 \%(n=16)$ of all studies nominated 'classic' rash distribution as part of the diagnosis, there was variation in the actual sites of rash used, with several studies not defining the relevant body locations, while other studies 
specified up to 15 sites ${ }^{25}$ Body diagrams, clinical photography and severity scales were used as adjuncts to clinical assessment in a number of trials; however, these tools were used as case or severity descriptors, rather than contributors to the diagnosis.

Although 45 trials $(63 \%)$ conducted parasitological tests, not all used this information for diagnosis (Table 3). Only $23(51 \%)$ of those that did perform testing required a positive test for diagnosis. Skin scrapings were the predominant form of testing specified in 39 trials $(87 \%)$, and only $8(18 \%)$ described their sample processing method.

In combining the diagnostic aspects used, 26 trials (37\%) specified both clinical and testing approaches in order to reach the diagnosis of scabies. Some trials specified only 'clinical examination' ( $n=14,20 \%)$, or 'testing' ( $n=14,20 \%)$ aspects. The remaining 17 trials (24\%) did not describe any specific clinical or testing aspects.

Of the 42 clinic-based trials, $27(64 \%)$ used parasitological testing as a component of diagnosis, compared with $9(39 \%)$ of 23 field trials $(P=0.07)$. A higher proportion of studies that involved all age groups $(60 \%)$ used testing compared with studies of children $(31 \% ; P=0.11)$. There was no difference in the proportion of trials in resource-limited and non-resource-limited countries that used testing (56\% versus $57 \%, P=0.94)$, nor was there a difference between studies published since $2000(61 \%)$ and those published earlier $(50 \%, P=0.47)$.

Only 10 studies $(14 \%)$ provided a reference for the diagnostic approach used. These included skin scraping methods, rash appearance in children, or broader descriptive references.

\section{Discussion}

In this systematic review of scabies therapeutic trials, we found that most studies used poorly defined diagnostic criteria. Nearly a quarter of trials did not describe any aspect of how the diagnosis of scabies was made. Slightly over half of the trials clarified their clinical diagnostic criteria, predominantly using rash, rash distribution, pruritus and the presence of mite burrows. Similarly, just over half of all of trials used parasitological testing for diagnostic purposes. Of the trials that did specify the features used for diagnostic decision-making, features were frequently described vaguely, prohibiting confident interpretation of the methods used. The wide variation in definitions of rash characteristics and distribution further impedes trial comparison.

No predominant combination of diagnostic criteria emerged through the review. It was often unclear whether all diagnostic features were required to meet case definition criteria or whether cases with fewer features were included. Likewise, where testing was used, it was often not performed for all subjects or was not a required feature for diagnosis.

Our findings are in keeping with those of Leung et al., whose review of the evidence behind diagnostic methods for scabies concluded that diagnosis is often 'imprecise and speculative'. ${ }^{26}$ Our study aldo overlaps with the 2007 Cochrane review of scabies treatment, ${ }^{27}$ which reported that all 22 included trials used a clinical diagnosis of scabies, but did not examine this diagnostic process. There was parasitological confirmation in $33.7 \%$ of included participants (903 of 2676), supporting our finding that this is not a consistent feature used for diagnosis.

The observed difference in diagnostic approaches in field versus clinic settings is in keeping with the pragmatics of available resources. This presents a challenge for guideline development, as a single approach may not suit each research or clinical environment. Diagnostic guidelines must be practical in a 
low-resource clinical setting, while maintaining diagnostic rigour. The development of point-of-care serological testing may improve diagnostic confirmation; however, such testing must be accurate, straightforward and cost-effective.

Our review is limited by including articles published in English only. Additionally, we only extracted diagnostic information that was clearly stated in the Methods sections of articles, and discounted suggested or implied terms mentioned elsewhere within the reports. Further, we reviewed therapeutic trials and did not include epidemiological studies. However, a recent a systematic review of scabies prevalence observed a similar lack of diagnostic clarity in included studies. ${ }^{5}$

The high burden of disease caused by scabies, ${ }^{5}$ the increasing recognition of its serious complications, ${ }^{1,28}$ and the availability of highly effective anti-scabetic therapies ${ }^{27,29}$ all provide impetus for the development of new control approaches. There is a pressing need for consensus diagnostic criteria to enable accurate comparison of epidemiological data and assessment of treatment methods. The development of diagnostic guidelines through a recognized consensus process has been initiated by the International Alliance for the Control of Scabies. ${ }^{1,5}$ These guidelines will provide a framework for collaboration and innovation as progress is made toward control of this debilitating disease.

\section{Acknowledgements}

We thank the British Association of Dermatologists (UK) and Diana Martin of the Centres for Disease Control (USA), for their assistance in sourcing the included articles. This study was supported by National Health \& Medical Research Council (Australia) Fellowship funding (to DE and AS).

\section{What is already known about this topic?}

- Scabies causes significant morbidity and affects marginalized groups worldwide.

- The clinical features of scabies overlap with those of other skin conditions, and pathognomonic features may not be present or easily demonstrable.

- Skin scrapings for scabies are prone to false negatives and not always practical, especially in resource-limited settings.

- There are no consensus guidelines for scabies, which limits the comparison and interpretation of existing epidemiological and intervention studies.

\section{What does this study add?}

- Published therapeutic trials for scabies have used variable diagnostic approaches.

- The clinical findings used for scabies diagnosis were often poorly described and included a wide range of features.

- Testing for scabies was variably applied.

- There were no consistent criteria used for diagnosis in the therapeutic trials for scabies.

- This review supports the need for the development of consensus scabies diagnostic guidelines to enable research aimed at controlling this disease.

\section{References}

1. Engelman D, Kiang $\mathrm{K}, \mathrm{Chosidow} \mathrm{O}$ et al. Toward the global control of human scabies: introducing the 
International Alliance for the Control of Scabies. PLoS Negl Trop Dis 2013; 7: e2167.

2. Vos T, Flaxman AD, Naghavi M et al. Years lived with disability (YLDs) for 1160 sequelae of 289 diseases and injuries 1990-2010: a systematic analysis for the Global Burden of Disease Study 2010. Lancet 2012; 380: 2163-96.

3. Heukelbach J, Feldmeier H. Scabies. Lancet 2006; 367: 1767-74.

4. World Health Organization (Department of Child and Adolescent Health and Development). Epidemiology and Management of Common Skin Diseases in Children in Developing Countries. Geneva: World Health Organization. 2005.

5. Romani L, Steer AC, Whitfeld MJ et al. Prevalence of scabies and impetigo worldwide: a systematic review. Lancet Infect Dis 2015; 15: 960-7.

6. Taplin D, Porcelain SL, Meinking TL et al. Community control of scabies: a model based on use of permethrin cream. Lancet 1991; 337: 1016-18.

7. Terry BC, Kanjah F, Sahr F et al. Sarcoptes scabiei infestation among children in a displacement camp in Sierra Leone. Public Health 2001; 115: 208-11.

8. Stanton B, Khanam S, Nazrul H et al. Scabies in urban Bangladesh. J Trop Med Hyg 1987; 90: 21926.

9. Jackson A, Heukelbach J, Filho AF et al. Clinical features and associated morbidity of scabies in a rural community in Alagoas, Brazil. Trop Med Int Health 2007; 12: 493-502.

10. Carapetis JR, Steer AC, Mulholland EK et al. The global burden of group A streptococcal diseases. Lancet Infect Dis 2005; 5: 685-94.

11. Lawrence G, Leafasia J, Sheridan J et al. Control of scabies, skin sores and haematuria in children in the Solomon Islands: Another role for ivermectin. Bull World Health Org 2005; 83: 34-42.

12. McDonald M, Currie BJ, Carapetis JR. Acute rheumatic fever: a chink in the chain that links the heart to the throat? Lancet Infect Dis 2004; 4: 240-5.

13. Roberts LJ, Huffam SE, Walton SF et al. Crusted scabies: clinical and immunological findings in seventy-eight patients and a review of the literature. $J$ Infect 2005; 50: 375-81.

14. Johnson CG, Mellanby K. The parasitology of human scabies. Parasitology 1942; 34: 285-90.

15. Bartley WC, Mellanby K. The parasitology of human scabies (women and children). Parasitology 1944; 35: 207-8.

16. Johnston G, Sladden M. Scabies: diagnosis and treatment. BMJ 2005; 331: 619-22.

17. Chouela E, Abeldano A, Pellerano $G$ et al. Diagnosis and treatment of scabies: a practical guide. Am J Clin Dermatol 2002; 3: 9-18.

18. Dupuy A, Dehen L, Bourrat E et al. Accuracy of standard dermoscopy for diagnosing scabies. $J$ Am Acad Dermatol 2007; 56: 53-62.

19. Argenziano G, Fabbrocini G, Delfino M. Epiluminescence microscopy. A new approach to in vivo detection of Sarcoptes scabiei. Arch Dermatol 1997; 133: 751-3.

20. Zheng Y, He R, He M et al. Characterization of Sarcoptes scabiei cofilin gene and assessment of recombinant cofilin protein as an antigen in indirect-ELISA for diagnosis. BMC Infect Dis 2016; 16: 21.

21. Mounsey KE, McCarthy JS, Walton SF. Scratching the itch: new tools to advance understanding of scabies. Trends Parasitol 2013; 29: 35-42.

22. United Nations Development Programme. Human Development Report. New York: United Nations Development Programme, 2013.

This article is protected by copyright. All rights reserved 
23. Moher D, Liberati A, Tetzlaff $\mathrm{J}$ et al. Preferred reporting items for systematic reviews and metaanalyses: the PRISMA statement. PLoS Med 2009; 6: e1000097.

24. Bañez JA, Nazareno RC, Medel RB. Clinical trial on the effectiveness of gliricidia sepium (kakawati) in treating patients with scabies in the antipolo CBHP. Phil J Microbiol Infect Dis 1999; 28: 115-324.

25. Castillo AL, Osi MO, Ramos JDA et al. Efficacy and safety of Tinospora cordifolia lotion in Sarcoptes scabiei var hominis-infected pediatric patients: A single blind, randomized controlled trial. $J$ Pharmacol Pharmacother 2013; 4: 39-46.

26. Leung V, Miller M. Detection of scabies: A systematic review of diagnostic methods. Can J Infect Dis Med Microbiol 2011; 22: 143-6.

27. Strong M, Johnstone PW. Interventions for treating scabies. Cochrane Database Syst Rev 2007; (3):CD000320.

28. Bowen AC, Mahe A, Hay RJ et al. The Global epidemiology of impetigo: a systematic review of the population prevalence of impetigo and pyoderma. PLoS One 2015; 10: e0136789.

30. Romani $L$, Whitfeld MJ, Koroivueta $\mathrm{J}$ et al. Mass drug administration for scabies control in a population with endemic disease. N Engl J Med 2015; 373: 2305-13.

Figure 1 Systematic review flowchart.n

Table 1 Trial setting and population characteristics.

\begin{tabular}{lrr}
\hline $\begin{array}{l}\text { Study characteristics } \\
(N=71)\end{array}$ & $n$ & $\%$ \\
\hline Location & 23 & 32 \\
Field & 42 & 59 \\
Clinic & 6 & 8 \\
Unspecified & & \\
Resource-limited & 53 & 75 \\
Country & 31 & 44 \\
Vulnerable group & 16 & 23 \\
Institution & & \\
Age group & 37 & 52 \\
All ages & 18 & 25 \\
Adults only & 13 & 18 \\
Children only & 3 & 4 \\
Unspecified & &
\end{tabular}

Table 2 Clinical features used to diagnose scabies.

\begin{tabular}{llll} 
& & \multicolumn{2}{l}{ Percentage of: } \\
\cline { 3 - 4 } & & & \multicolumn{1}{c}{$\begin{array}{l}\text { trials using } \\
\text { clinical }\end{array}$} \\
& & all trials & examination \\
Clinical feature & $n$ & $(N=71)$ & $(N=40)$ \\
\hline Unspecified clinical examination* & 31 & 44 & -
\end{tabular}

This article is protected by copyright. All rights reserved 
Pruritus

$\begin{array}{llll}\text { Any } & 28 & 39 & 70 \\ \text { Nocturnal } & 18 & 25 & 45\end{array}$

Rash

$\begin{array}{lrrr}\text { Any } & 40 & 56 & 100 \\ \text { Burrows } & 22 & 31 & 55 \\ \text { Papules } & 20 & 28 & 50 \\ \text { Nodules } & 9 & 13 & 23 \\ \text { Vesicles } & 12 & 17 & 30 \\ \text { Pustules } & 8 & 11 & 20 \\ \text { Excoriation } & 6 & 8 & 15\end{array}$

Rash distribution

\begin{tabular}{lrrr}
\multicolumn{1}{c}{ Any } & 30 & 42 & 75 \\
Classic & 16 & 23 & 40 \\
Contact history & 16 & 23 & 40 \\
Magnification & 5 & 7 & 13
\end{tabular}

${ }^{*}$ Refers to articles that did not provide any details regarding clinical examination findings used to diagnose scabies.

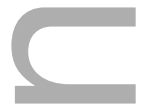

Table 3 Testing approaches used.

\begin{tabular}{|c|c|c|c|}
\hline \multirow[b]{2}{*}{ Testing approach } & \multirow[b]{2}{*}{$n$} & \multicolumn{2}{|c|}{ Percentage of: } \\
\hline & & $\begin{array}{l}\text { all trials } \\
(N=71)\end{array}$ & $\begin{array}{l}\text { trials } \\
\text { using } \\
\text { testing } \\
(N=45)\end{array}$ \\
\hline Any testing for parasite & 45 & 63 & - \\
\hline Testing for diagnostic purposes & 40 & 56 & 89 \\
\hline $\begin{array}{l}\text { Positive test required for diagnosis } \\
\text { Skin scraping }\end{array}$ & 23 & 32 & 51 \\
\hline Performed $^{*}$ & 39 & 55 & 87 \\
\hline Processing method $\dagger$ & 8 & 11 & 18 \\
\hline Mite identification $\ddagger$ & 38 & 54 & 84 \\
\hline Mite product§ & 27 & 38 & 60 \\
\hline
\end{tabular}

${ }^{\star}$ A skin scraping was specified as the type of test performed; $\uparrow$ indicates that the report described that trial's method of processing the skin scraping sample; fadult or larval mite forms were accepted for a positive skin scraping result; §ova or scybala were accepted for a positive skin scraping result.

\section{CPD questions}

\section{Learning objective:}

To appreciate the challenges in diagnosing scabies, in the context of the epidemiology, complications and 
clinical signs of the disease.

\section{Question 1}

In which of the following groups is scabies most prevalent?
(a) Young children.
(b) Adolescents.
(c) Adults.
(d) Elderly people.
(e) People with suppressed immunity.

\section{Question 2}

Which of the following is the most specific test for scabies?
(a) Clinical examination.
(b) Serology
(c) PCR.
(d) Dermoscopy.
(e) Microscopy of skin scrapings.

\section{Question 3}

The complications of scabies can include which of the following?
(a) Impetigo
(b) Invasive bacterial disease.
(c) Local abscess.
(d) Post-streptococcal glomerulonephritis.
(e) All of the above.

\section{Question 4}

In this study, which of the following were the predominant clinical criteria for diagnosis of scabies?
(a) Rash and pruritus.
(b) Rash, pruritus and burrows.
(c) Pruritus and positive skin scrapings.
(d) Pruritus that is worse at night and an affected family member.
(e) There were no predominant clinical criteria found.

\section{Question 5}

Which of the following clinical findings is pathognomonic of scabies?
(a) Axillary nodules.
(b) Papules in finger web spaces.
(c) Papules on palms of infants.
(d) Pruritic papules on the buttocks.
(e) Burrows 


\section{Answer 1}

In which of the following groups is scabies most prevalent?

(a) Correct. Children have the highest prevalence of scabies in most settings.

(b) Incorrect. Although adolescents have a high prevalence, the prevalence in most settings is not highest in this group.

(c) Incorrect. The prevalence in most settings is not highest in this group.

(d) Incorrect. The prevalence in most settings is not highest in this group.

(e) Incorrect. Although people with suppressed immunity may be at risk of crusted scabies in particular, the prevalence of scabies in most settings is not highest in this group.

\section{Answer 2}

Which of the following is the most specific test for scabies?

(a) Incorrect. Clinical examination is not the most specific test.

(b) Incorrect. There are no serological tests for scabies currently available.

(c) Incorrect. There are no nucleic acid-based tests for scabies currently available.

(d) Incorrect. Although dermoscopy may be helpful for diagnosis, it is not the most specific test.

(e) Correct. Microscopy of skin scrapings allows direct visualisation of the mite and confirms the diagnosis.

\section{Answer 3}

The complications of scabies can include which of the following?
(a) Incorrect.
(b) Incorrect.
(c) Incorrect.
(d) Incorrect.
(e) Correct. All are known complications of scabies.

\section{Answer 4}

In this study, which of the following were the predominant clinical criteria for diagnosis of scabies?
(a) Incorrect
(b) Incorrect.
(c) Incorrect.
(d) Incorrect.
(e) Correct. There were no predominant clinical criteria found.

\section{Answer 5}

Which of the following clinical findings is pathognomonic of scabies?
(a) Incorrect. Axillary nodules may be seen, particularly in children, but are not specific to scabies.
(b) Incorrect. Web space papules are suggestive but not pathognomonic.
(c) Incorrect. Other conditions such as infantile acropustulisis can cause this.
(d) Incorrect. Papules on the buttocks can be seen in several conditions.
(e) Correct. Burrows are pathognomonic of scabies infestation. 


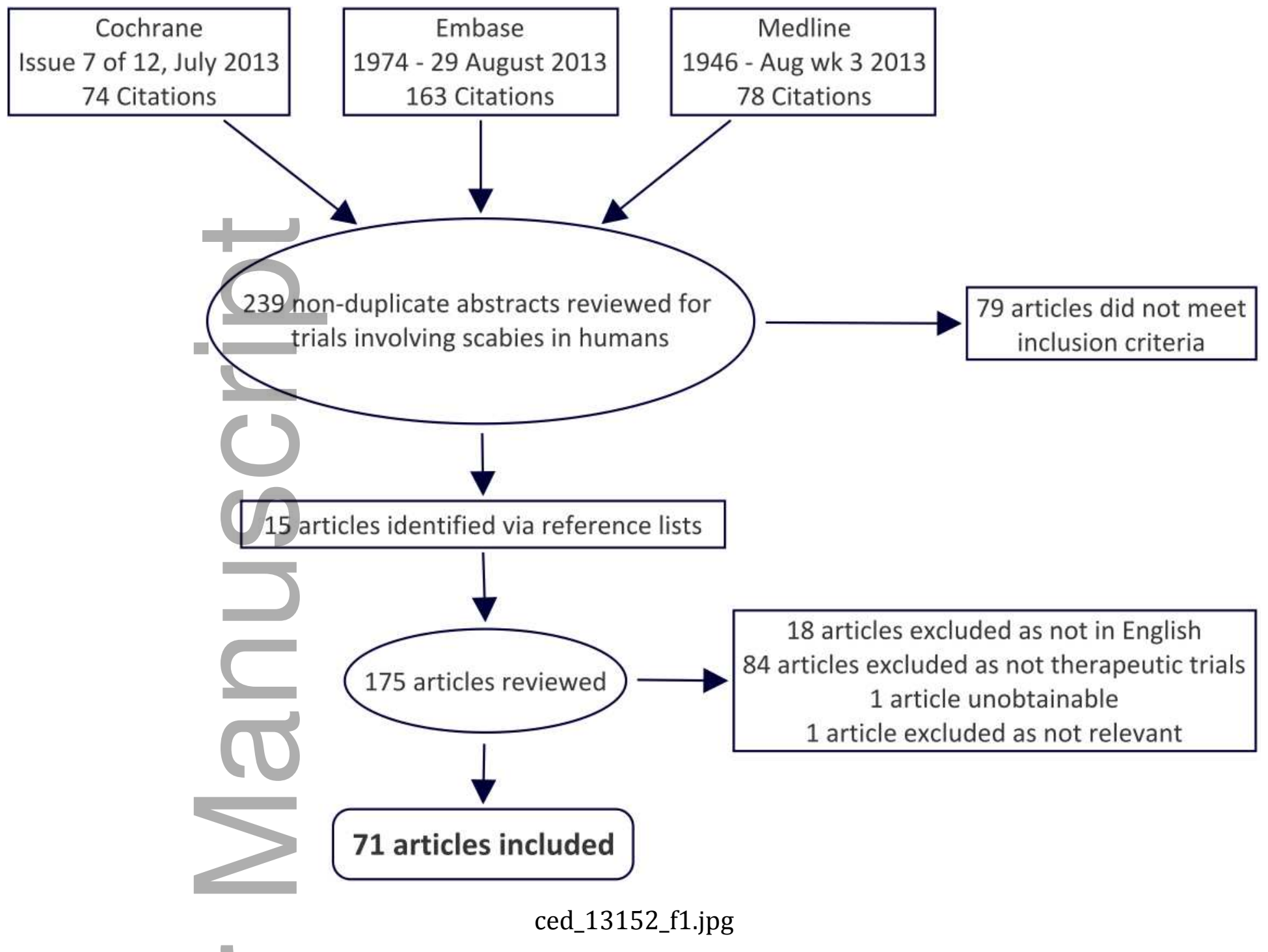

This article is protected by copyright. All rights reserved 


\section{University Library}

\section{- M M N E R VA A gateway to Melbourne's research publications}

Minerva Access is the Institutional Repository of The University of Melbourne

Author/s:

Thompson, MJ;Engelman, D;Gholam, K;Fuller, LC;Steer, AC

Title:

Systematic review of the diagnosis of scabies in therapeutic trials

Date:

2017-07-01

Citation:

Thompson, M. J., Engelman, D., Gholam, K., Fuller, L. C. \& Steer, A. C. (2017). Systematic review of the diagnosis of scabies in therapeutic trials. CLINICAL AND EXPERIMENTAL DERMATOLOGY, 42 (5), pp.481-487. https://doi.org/10.1111/ced.13152.

Persistent Link:

http://hdl.handle.net/11343/292949 